\title{
Ferroelectricity and tunneling electroresistance effect driven by asymmetric polar interfaces in all-oxide ferroelectric tunnel junctions
}

\author{
L. L. Tao and J. Wang ${ }^{\text {a) }}$ \\ Department of Physics and the Center of Theoretical and Computational Physics, \\ The University of Hong Kong, Hong Kong, China
}

(Received 17 December 2015; accepted 30 January 2016; published online 12 February 2016)

\begin{abstract}
By constructing asymmetric polar interfaces, all-oxide ferroelectric tunnel junctions (FTJs) are proposed that can achieve a sizable tunneling electroresistance (TER) effect. Based on first-principles quantum transport calculations on a prototypical $\mathrm{LaNiO}_{3} / \mathrm{BaTiO}_{3} / \mathrm{LaNiO}_{3}$ junction, we predict that TER reaches $10^{3} \%$ under a finite bias. Driven by the asymmetric polar interfaces, the resultant intrinsic electric field causes a highly asymmetric electrostatic potential in comparison to that of the FTJ with symmetric polar interfaces. As a result, the tunneling resistance changes significantly upon polarization reversal leading to sizable TER. The physical origin of the TER effect can be well understood in terms of local density of states, transport in momentum space, real-space scattering states and a free-electron tunneling model. Our results provide an insight into the understanding of ferroelectricity and the TER mechanism in FTJs and will be useful for FTJ-based devices design. (C) 2016 AIP Publishing LLC. [http://dx.doi.org/10.1063/1.4941805]
\end{abstract}

Recently there has been an increasing interest in ferroelectric tunnel junctions (FTJs) because of the rich physics they display as well as their potential applications in electronic devices. ${ }^{1}$ A typical FTJ consists of two metal electrodes separated by a thin ferroelectric (FE) film. ${ }^{2}$ Due to the advancement of nanotechnology, a stable polarization can be maintained in a few nm thin FE film. ${ }^{3-5}$ As the electron tunnels through the FE barrier, the tunneling resistance can be changed with the polarization reversal, which in turn gives rise to the well-known tunneling electroresistance (TER) effect. So far a number of approaches have been proposed to achieve a sizable TER, such as constructing FTJs with asymmetric electrodes, ${ }^{6,7}$ using the piezoelectric effect of FE that modulates the barrier width, ${ }^{7,8}$ constructing non-equivalent interfaces, ${ }^{9}$ intercalating a nonpolar dielectric layer at the interface, ${ }^{10,11}$ using doped manganite electrodes whose magnetic order at the interface can be switchable by the polarization, ${ }^{12,13}$ and using $n$-type semiconductor electrodes. ${ }^{14,15}$ Here, we propose a different approach to achieve a sizable TER effect in all-oxide FTJs: through constructing asymmetric polar interfaces, a formed intrinsic electric field results in rather different tunneling barrier heights for different polarization orientations leading to a sizable TER effect.

To illustrate our idea, we consider a FTJ made of an all-oxide $\mathrm{LaNiO}_{3} / \mathrm{BaTiO}_{3} / \mathrm{LaNiO}_{3}$ junction with left interface termination of $\left(\mathrm{NiO}_{2}\right)^{-} /(\mathrm{BaO})^{0}$ type and of the $\left(\mathrm{LaO}_{2}\right)^{+} /$ $\left(\mathrm{TiO}_{2}\right)^{0}$ type for the right interface, as shown in Fig. 1(a). Recently, epitaxial $\mathrm{LaNiO}_{3} / \mathrm{BaTiO}_{3}$ heterostructures maintaining a stable polarization have been fabricated, ${ }^{16}$ suggesting that our proposed FTJ structure is experimentally feasible. $\mathrm{LaNiO}_{3}$ (LNO) is a polar perovskite oxide which consists of the alternating positively charged $\left(\mathrm{LaO}_{2}\right)^{+}$ layer and negatively charged $\left(\mathrm{NiO}_{2}\right)^{-}$layer, which is rather similar to the perovskite oxide $\mathrm{LaAlO}_{3}$. As such, LNO can directly support electron ( $\mathrm{LaO}$ termination) or hole ( $\mathrm{NiO}$

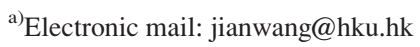

termination) doping at the interface when it is deposited on the non-polar oxides (e.g., $\mathrm{SrTiO}_{3}$ or $\mathrm{BaTiO}_{3}$ ) via electronic reconstruction to avoid the electrostatic potential divergence. ${ }^{17}$ Due to the asymmetric polar interfaces, an intrinsic electric field $E_{i}$ is directly formed within the $\mathrm{BaTiO}_{3}$ (BTO) film, as schematically shown in Fig. 1(a). Moreover, in contrast to insulator oxide $\mathrm{LaAlO}_{3}$, LNO is a good conductive oxide and can thus be used as electrodes for many devices. ${ }^{16}$ In parallel, perovskite oxide BTO is a widely used ferroelectric material, exhibiting large polarization as well as a high Curie temperature of $\sim 120^{\circ} \mathrm{C}$. It was shown that a nonswitchable ferroelectric dead layer can be formed near the interface due to the $E_{i}$ created by a polar interface. ${ }^{18}$ It was also demonstrated that the ferroelectricity in LNO/BTO

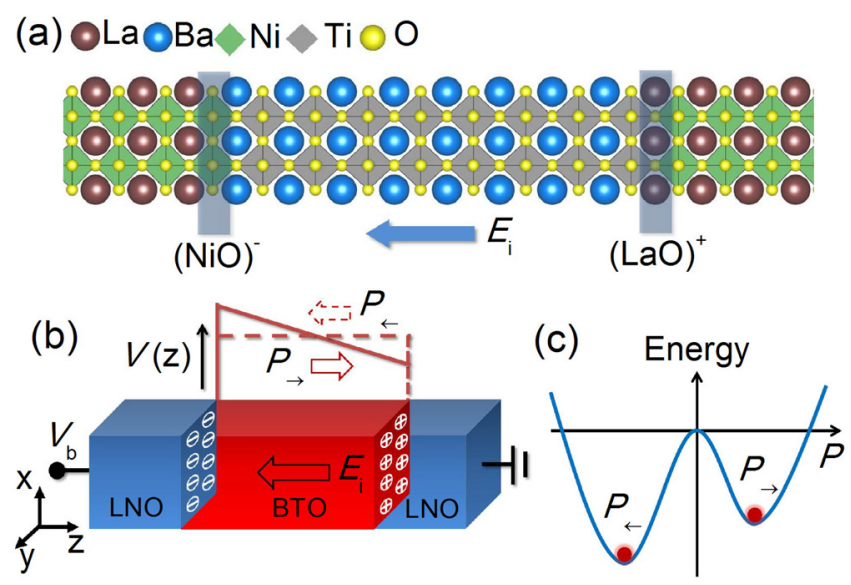

FIG. 1. (a) Atomic structure of LNO/BTO/LNO FTJ with the $\mathrm{NiO} / \mathrm{BaO}$ termination at the left interface and $\mathrm{LaO} / \mathrm{TiO}$ at the right interface. The intrinsic electric field $E_{i}$ is pointing from $(\mathrm{LaO})^{+}$interface to $(\mathrm{NiO})^{-}$interface. (b) A schematic FTJ device model and the potential profile $V(z)$ for left polarization $\left(P_{\leftarrow}\right.$, dashed line) and right polarization $\left(P_{\rightarrow}\right.$, solid line). The symbols $\oplus$ and $\ominus$ denote the positively charged $(\mathrm{LaO})^{+}$layer and negatively charged $(\mathrm{NiO})^{-}$layer, respectively. $V_{b}$ is the applied bias. (c) Schematic double well potential for the FTJ, that is, the potential energy as a function of polarization $P$. 
superlattices is associated with polar interface type due to the different penetration lengths between electron or hole. ${ }^{19}$ These previous works indicate that an intrinsic electric field can be created by the polar interface, and more importantly, such an intrinsic electric field may affect the ferroelectricity of the FE film significantly. This motivates us to explore the possibility of achieving a sizable TER effect for a FTJ with asymmetric polar interfaces. By first-principles calculations on the LNO/BTO/LNO FTJs, we investigate the ferroelectricity in such a junction and predict a giant TER effect of upto $10^{3} \%$ under a finite bias. Note that the atomic and electronic structures were calculated using the DFT-based VASP electronic package ${ }^{20}$ and quantum transport calculations are performed using a Keldysh-nonequilibrium-Green's-function (NEGF)-based Nanodcal package. ${ }^{21}$ All the computational details are reported in the supplementary material. ${ }^{22}$

First we study ferroelectric stability for a series of FTJs as shown in Fig. 1(a). The supercell is denoted as (LaO$\mathrm{NiO})_{7} /(\mathrm{BaO}-\mathrm{TiO})_{m}$, where $m=2-10$. Fig. 2(a) shows relative metal-O (M-O) displacements in the BTO film for either left (negative displacements) or right (positive displacements) polarization state. Note that such left/right polarization state was obtained by fully relaxing the atomic positions starting from the artificially left/right ferroelectric configuration. It is intriguing to see that there is always a nonzero M-O displacement even for only one-unit-cell $(m=2)$ thick of BTO film, indicating the vanishing of ferroelectric critical thickness. This is due to the fact that $E_{i}$ always enforces the $\mathrm{M}-\mathrm{O}$ displacements. On the other hand, only the left polarization states are stable when $m$ is smaller than 8 , as evident from the only negative $\mathrm{M}-\mathrm{O}$ displacements in the inset of
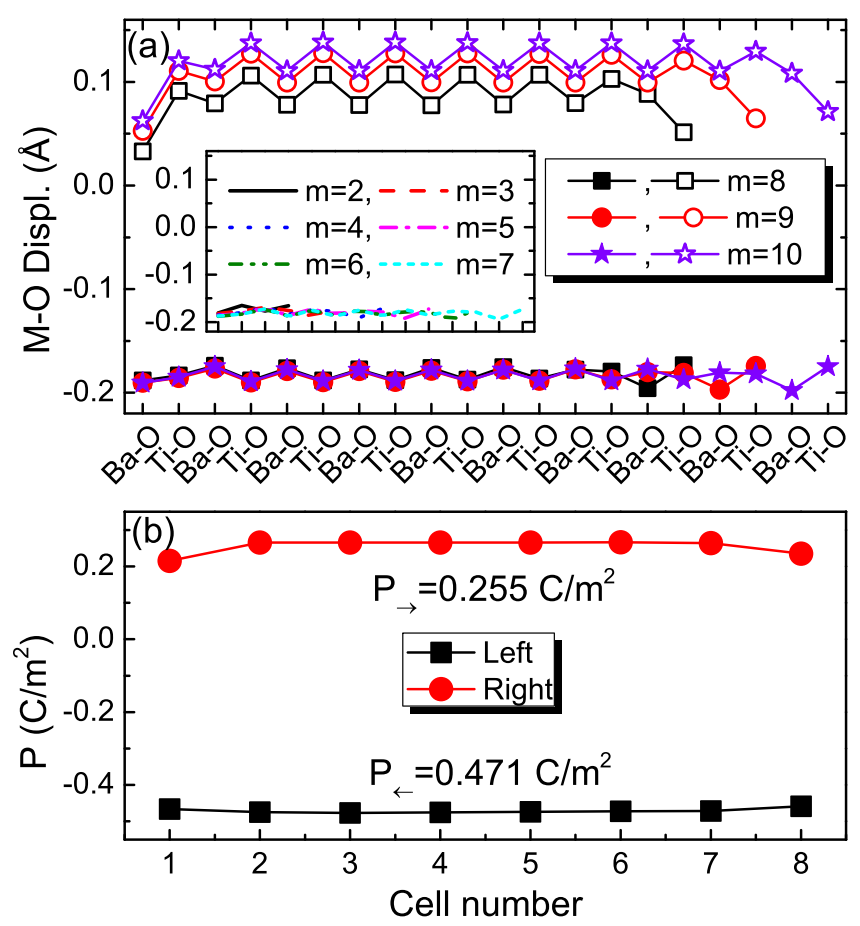

FIG. 2. (a) The relative metal-O (M-O) displacements in the BTO film for left (solid symbols) and right (open symbols) polarization states for different thickness $m=8-10$. Inset: the similar M-O displacements in BTO film with thickness $m=2-7$. (b) Local polarization $P$ mapped onto unit cell for the BTO film with thickness $m=8$. The averaged polarization $P_{\leftarrow, \rightarrow}$ for left (squares, $\leftarrow$ ) and right (circles, $\rightarrow$ ) polarization states is marked.
Fig. 2(a). For the cases of $m=8-10$, both the left and right polarization states are stable and M-O displacements are uniform through the BTO film. In addition, the displacements for left polarization are slightly larger than that for right polarization. For example, in the case of $m=8$, the Ti-O displacements in the central $\mathrm{TiO}$ layers are about $0.19 \AA$ and $0.11 \AA$ for left and right polarizations, respectively. ${ }^{23}$ From those M-O displacements and the Born effective charges, one can evaluate the local polarization $P(k)$ by $^{24}$

$$
P(k)=\frac{1}{\Omega_{k}} \sum_{m} Z_{m}^{*} \delta z_{m},
$$

where $P(k)$ is the polarization of the $k$ th unit cell whose volume is $\Omega_{k}, Z_{m}^{*}$ the Born effective charge of the $m$ th atom, and $\delta z_{m}$ the displacement of the $m$ th atom. The Born effective charges $Z_{m}^{*}$ are calculated using density functional perturbation theory ${ }^{25}$ for $\mathrm{Ba}, \mathrm{Ti}, \mathrm{O}_{\perp}\left(\mathrm{TiO}\right.$ plane) and $\mathrm{O}_{\|}(\mathrm{BaO}$ plane) ions are respectively $2.70,6.66,-2.07$, and -5.20 electrons. The local polarization across BTO film for $m=8$ case is shown in Fig. 2(b). One can see that the magnitude of local polarization is consistent with that of $\mathrm{M}-\mathrm{O}$ displacement for the $m=8$ case shown in Fig. 2(a). As discussed below, such asymmetric polarizations result in a rather different potential profile which is responsible for the TER effect, as schematically plotted in Fig. 1(b). The corresponding schematic double-well potential is plotted in Fig. 1(c). To confirm this Fig. S2 shows the electrostatic potential profiles for two distinct polarization orientations. Comparing Figs. S2(a) and S2(b) with Fig. S2(c) (see solid lines), it is clear that the potential profile for the asymmetric FTJs is more asymmetric than that of symmetric FTJs.

Having investigated the polarization stability of FTJs, we analyze the electronic structures through the layer-resolved local density of states (LDOS) for the two polarization states, which is plotted in Fig. 3. It is seen that the position of conduction-band minimum (CBM) is roughly constant through the BTO film for the left polarization state [see Fig. $3(a)$ ], indicating that the effective electric field is small. In contrast, there is an obvious variation in CBM through the BTO film for the right polarization state [see Fig. 3(b)], indicating that a sizable electric field exists that shifts the VBM rigidly. This is expected because the effective electric filed is the combination of depolarization field $E_{d}$ resulting from an incomplete screening by the electrodes and an intrinsic field $E_{i}$ resulting from an asymmetric polar interface. The direction of $E_{d}$ is associated with polarization orientation and thus $E_{d}$ and $E_{i}$ have the same (opposite) direction for the right (left) polarization. Consequently, the effective electric field is enhanced (reduced) for right (left) polarization, thus resulting in different CBM-variation tendency.

We now consider the TER effect by performing quantum transport calculations. The zero-bias conductance $G$ is given by the Landauer-Büttiker formula

$$
G=\frac{2 e^{2}}{h} \sum_{\mathbf{k}_{\|}} T\left(\mathbf{k}_{\|}, E_{F}\right)
$$

where $T\left(\mathbf{k}_{\|}, E_{F}\right)$ is the transmission coefficient of an electron at the Fermi energy $E_{F}$ with a transverse Bloch wave vector 


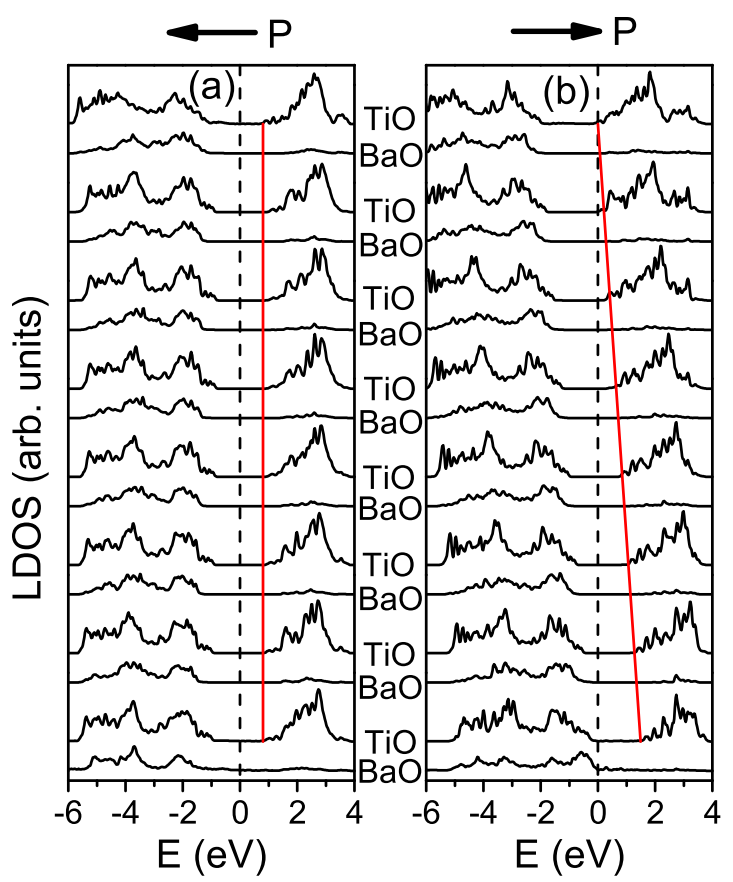

FIG. 3. Layer-resolved local density of states (LDOS) for (a) left and (b) right polarization states as denoted by the arrows. From bottom to top for either panel corresponds from the left interface $(\mathrm{NiO} / \mathrm{BaO}$ interface) to right interface [TiO/LaO interface, see Fig. 1(a)]. The vertical dashed lines denote the position of Fermi energy. The red solid lines denote roughly the variation tendency of layer-resolved position of conduction-band minimum (to guide our eyes).

$\mathbf{k}_{\|}=\left(k_{x}, k_{y}\right), e$ is the electron charge, and $h$ is the Planck's constant.

Our TER effect shall be quantified as TER $=\left(G_{\rightarrow}-\right.$ $\left.G_{\leftarrow}\right) / G_{\leftarrow}$, with $G_{\leftarrow}\left(G_{\rightarrow}\right)$ being the total tunneling conductance for a FTJ in left (right) polarization. Firstly we will focus on the first-principles results in zero-bias limit and the bias dependency of transport results will be discussed finally. We find that $G_{\rightarrow}$ is about five times larger than $G_{\leftarrow}$ and, as a consequence, large TER ratio of $\sim 450 \%$ is observed. As a comparison, Velev et al. predicted a zero-bias TER effect of $210 \%$ in all-oxide $\mathrm{SrRuO}_{3} / \mathrm{BaTiO}_{3} / \mathrm{SrRuO}_{3}$ FTJs with asymmetric non polar interfaces. Our obtained zero-bias TER effect is comparable to that reported in other all-oxide FTJs. ${ }^{9,11}$ To understand such a large TER effect, according to Eq. (2), it is helpful to examine the distribution of transmission coefficients in the two-dimensional Brillouin zone (BZ). As can be seen from Figs. 4(b) and 4(c), transmission is mainly contributed by the region around the center of BZ originating from the slow decay through the $\Delta_{5}$ symmetry state. Moreover, the shape of transmission distributions is rather similar to that of $\mathrm{La}_{1-x} \mathrm{Sr}_{x} \mathrm{MnO}_{3} / \mathrm{BTO}$ junctions ${ }^{12}$ and the gray areas showing zero transmission are consistent with the boundary of the Fermi surfaces of metal electrodes, which can be confirmed from Fig. 4(a). These are the incoming channels at the Fermi energy in the cross-sectional area of the LNO electrodes. As expected, there is an exactly oneto-one mapping for the gray positions between Figs. 4(a) and 4(b) or Figs. 4(a) and 4(c). On the other hand, by comparing Figs. 4(b) and 4(c), the transmission in left polarization is significantly reduced compared with the case of right polarization (note the different scale used in the sidebar on the right). Accordingly, it yields a significant total conductance reduction as the polarization changes from right to left giving rise to a large TER effect. ${ }^{26}$

To gain further insight, we analyze the TER mechanism from the viewpoint of scattering states. Scattering states $\psi_{s}$ are eigenstates of the open two-terminal device structure linking $z=-\infty$ to $z=+\infty,{ }^{21}$ which is quite useful to analyze transport properties. We plot the decay of the modulus of scattering states $\left|\psi_{s}(z)\right|$ at $\mathbf{k}_{\|}=(0,0)$ point through the junction for different polarization states in Fig. 5(a). It can be noticed that $\left|\psi_{s}(z)\right|$ decays exponentially through the BTO barrier for both polarization states, however the decay rate (the slope of the $\left|\psi_{s}(z)\right|$ ) for left polarization state is larger than that of the right polarization state. Consequently, a factor of about five differences in the magnitude of $\left|\psi_{s}(z)\right|$ can be found after tunneling through the barrier, in agreement with the TER data. Fig. 5(b) shows the real-space distribution of $\left|\psi_{s}(z)\right|$ across the junction for these two polarization states as denoted by the arrows. We find that the effective transmission channels are mainly composed of $\mathrm{Ti}$ (Ni)- $d$ orbital and O- $p$ orbital and $\left|\psi_{s}(z)\right|$ within the BTO is strongly suppressed upon polarization reversal from right to left as expected.

We now explain these results using a free-electron tunneling model, which is detailed in the supplementary material. $^{22}$ The inset of Fig. 6(b) shows the effective potential profile $V(z)$. We see that the slope of $V(z)$ for the right polarization state is larger than that for the left polarization state, indicating a larger effective electric field for the right polarization case as mentioned above. More interestingly, the
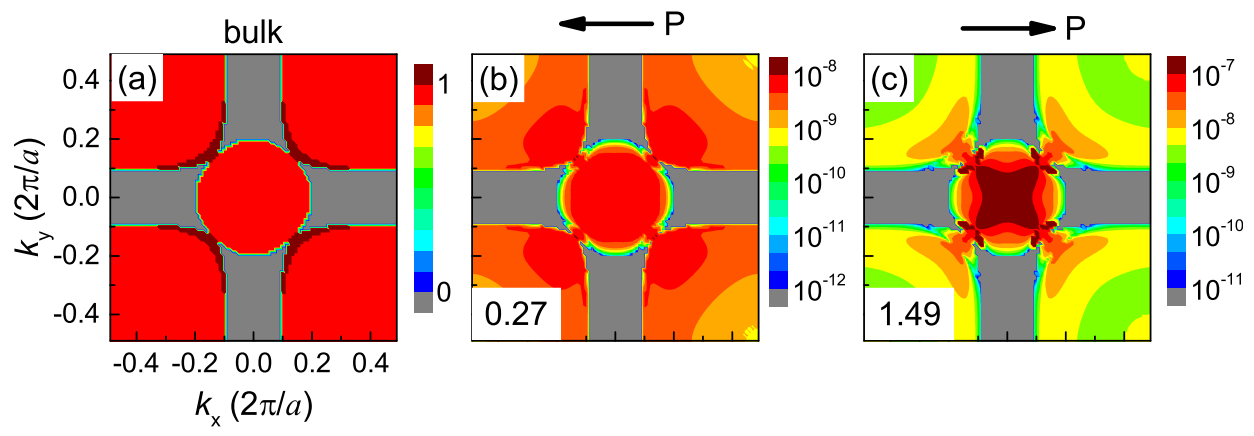

FIG. 4. (a): The $k_{\|}$-resolved transmission channels for bulk LNO at the Fermi energy. (b) and (c): the $k_{\|}$-resolved transmission coefficients at the Fermi energy of a FTJ: (b) for left polarization and (c) for right polarization states as denoted by the arrows. Note the different logarithmic scales for (b) and (c) were used in the side bars on the right. The numbers in the lower-left corner of (b) and (c) denote the integrated total conductance (in units of $10^{-8} e^{2} / h$ ) of each polarization state. 

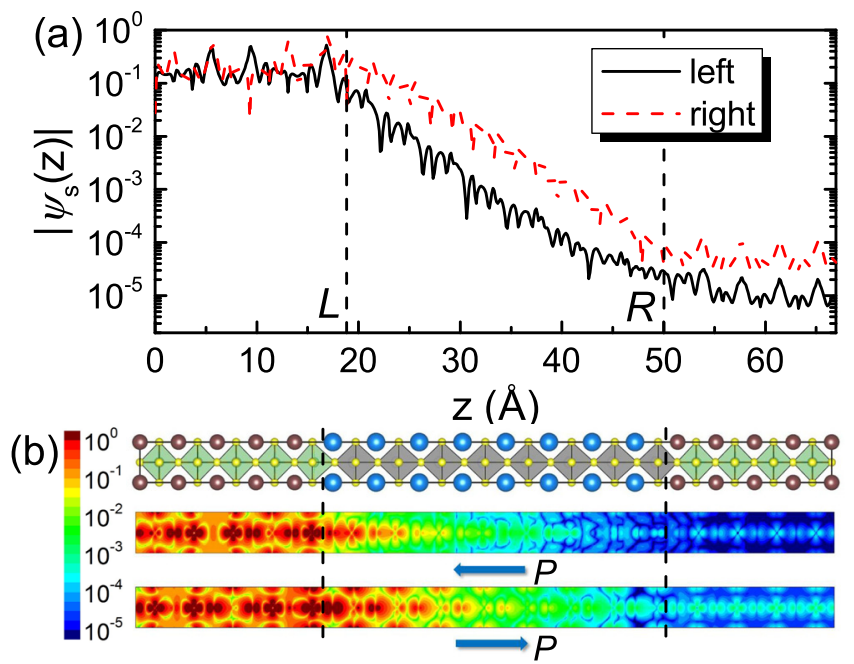

FIG. 5. (a) Distribution of the modulus of scattering states $\left|\psi_{s}(z)\right|$ at $\mathbf{k}_{\|}$ $=(0,0)$ point through the junction for left (black solid lines) and right (red dashed lines) polarization states. The two vertical dashed lines denote roughly the positions of left $(L)$ and right $(R)$ interfaces. (b) Real-space distribution of the modulus of $\left|\psi_{s}(z)\right|$ through the junction for left and right polarization states as denoted by the arrows. The side bar with logarithmic scale on the left represents the color coding for the modulus of $\left|\psi_{s}(z)\right|$.

shape of $V(z)$ is consistent with that of layer-resolved CBM as shown in Fig. 3 (see the solid red lines). On the other hand, the thickness of BTO barrier $(m=8)$ in our DFT calculations is about $30 \AA$. Considering the fact that there exists the metal-induced gap states (MIGS) in BTO for a few layers from the interface, ${ }^{27}$ the electron is not in tunneling regime through those MIGS, it is therefore somewhat less straightforward to determine the barrier thickness $d$ for a free-
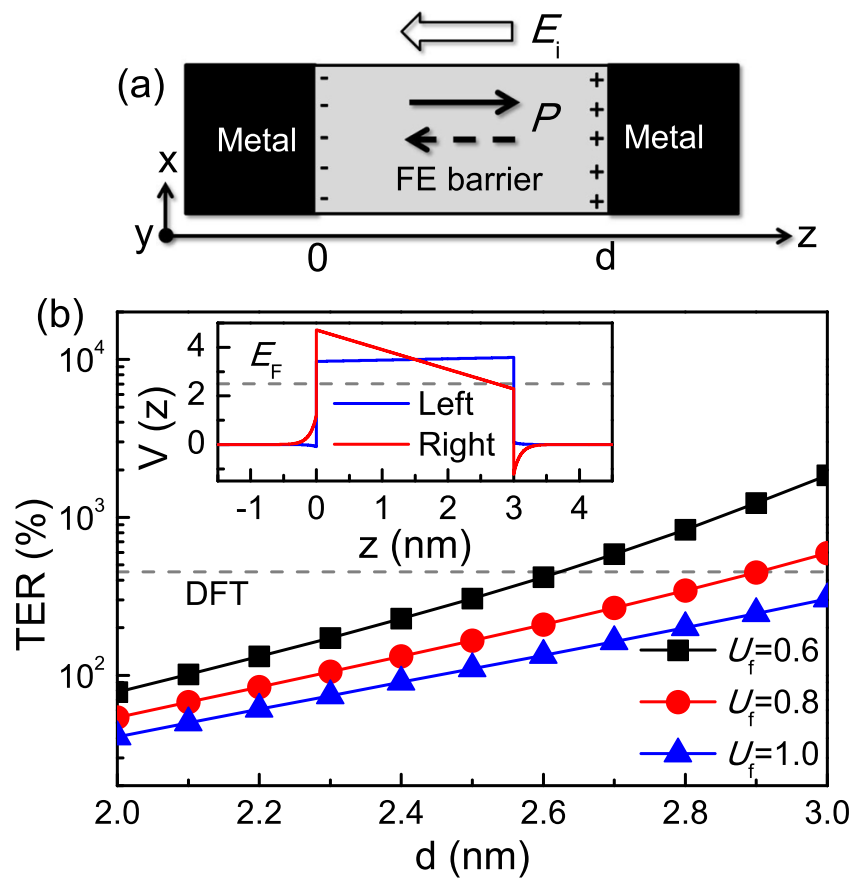

FIG. 6. (a) Sketch of a FTJ for two polarization states under short circuit boundary conditions. $P$ and $d$ stand for polarization and barrier thickness, respectively. The intrinsic filed $E_{i}$ pointing from right to left is denoted by the opened arrows. The symbols + and - stand for the ion charges in the polar layer. (b) TER as the function of barrier thickness $d$ for different barrier height $U_{f}$. The dashed line indicates the DFT result. Inset: Effective potential profile $V(z)$ of a FTJ for left (blue) and right (red) polarization states. The dashed line denotes the position of Fermi energy.

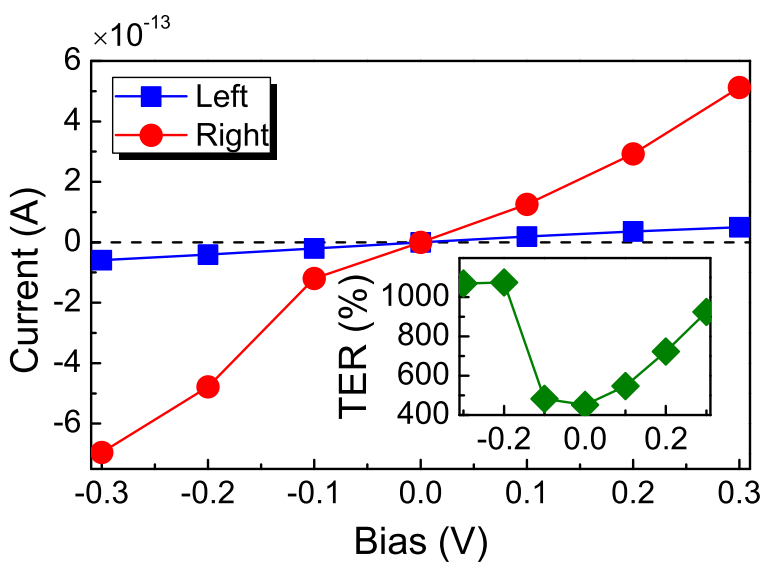

FIG. 7. Current as a function of bias for a FTJ in left and right polarization states. The inset shows the bias dependence of TER.

electron tunneling model. Therefore, we calculated the TER as a function of $d$ for a different barrier height $U_{f}$ in Fig. 6(b). We see that the TER ratio increases exponentially with the barrier thickness $d$, and the lower barrier height $U_{f}$ enhances the TER ratio.

Finally, we investigate the bias-dependent transport properties and TER effect by performing nonequilibrium quantum transport calculations. Fig. 7 shows the current-bias curves as well as the bias dependence of TER (inset) defined as TER $=\left(I_{\rightarrow}-I_{\leftarrow}\right) / I_{\leftarrow}$, with $I_{\leftarrow}\left(I_{\rightarrow}\right)$ being the total charge current for a FTJ in left (right) polarization. The polarity of bias $V_{b}$ is in accordance with the definition in Fig. 1(b). We define the zero-bias TER by using the transmission coefficient. The most striking feature is that there is a sizable difference between $I_{\rightarrow}$ and $I_{\leftarrow}$ and the TER reaches $\sim 10^{3} \%$ at a bias of $-0.2 \mathrm{~V}{ }^{28}$ This finding suggests that the asymmetry of the barrier can be greatly enhanced by the applied bias. In addition, the current-bias and TER curves are a little asymmetric with respect to the polarity of bias as expected from the asymmetric structure of our FTJ.

In summary, based on first-principles calculations, we have shown that sizable TER effects can be achieved in LNO/ $\mathrm{BTO} / \mathrm{LNO}$ with asymmetric polar interfaces due to the highly asymmetric tunneling potential caused by the intrinsic field. The obtained results were confirmed from an analysis of asymmetric ferroelectricity, local density of states, transmission in moment space and the decay of real-space scattering states. In addition, we have proposed a free-electron tunneling model that explains the TER effects very well. As such, we hope that our findings can stimulate the future experimental research and provide new insights into the designing of FTJ-based devices.

This work is supported by the University Grant Council of Hong Kong (Contract No. AoE/P-04/08). Atomic structure visualisation was produced with VESTA software. ${ }^{29} \mathrm{We}$ are grateful to the National Supercomputer Center in Tianjin for providing the computational facility.

${ }^{1}$ V. Garcia and M. Bibes, Nat. Commun. 5, 4289 (2014).

${ }^{2}$ E. Y. Tsymbal and H. Kohlstedt, Science 313, 181 (2006).

${ }^{3}$ T. Tybell, C. H. Ahn, and J.-M. Triscone, Appl. Phys. Lett. 75, 856 (1999).

${ }^{4}$ D. D. Fong, G. B. Stephenson, S. K. Streiffer, J. A. Eastman, O. Auciello, P. H. Fuoss, and C. Thompson, Science 304, 1650 (2004). 
${ }^{5}$ V. Garcia, S. Fusil, K. Bouzehouane, S. Enouz-Vedrenne, N. D. Mathur, A. Barthelemy, and M. Bibes, Nature 460, 81 (2009).

${ }^{6}$ M. Ye. Zhuravlev, R. F. Sabirianov, S. S. Jaswal, and E. Y. Tsymbal, Phys. Rev. Lett. 94, 246802 (2005).

${ }^{7}$ H. Kohlstedt, N. A. Pertsev, J. R. Contreras, and R. Waser, Phys. Rev. B 72, 125341 (2005).

${ }^{8}$ D. I. Bilc, F. D. Novaes, J. Iniguez, P. Ordejon, and P. Ghosez, ACS Nano 6, 1473 (2012).

${ }^{9}$ J. P. Velev, C.-G. Duan, J. D. Burton, A. Smogunov, M. K. Niranjan, E. Tosatti, S. S. Jaswal, and E. Y. Tsymbal, Nano Lett. 9, 427 (2009).

${ }^{10}$ M. Ye. Zhuravlev, Y. Wang, S. Maekawa, and E. Y. Tsymbal, Appl. Phys. Lett. 95, 052902 (2009).

${ }^{11}$ N. M. Caffrey, T. Archer, I. Rungger, and S. Sanvito, Phys. Rev. Lett. 109, 226803 (2012).

${ }^{12}$ J. D. Burton and E. Y. Tsymbal, Phys. Rev. Lett. 106, 157203 (2011).

${ }^{13}$ Y. W. Yin, J. D. Burton, Y-M. Kim, A. Y. Borisevich, S. J. Pennycook, S. M. Yang, T. W. Noh, A. Gruverman, X. G. Li, E. Y. Tsymbal, and Q. Li, Nat. Mater. 12, 397 (2013).

${ }^{14}$ Z. Wen, C. Li, D. Wu, A. Li, and N. Ming, Nat. Mater. 12, 617 (2013).

${ }^{15}$ Z. Wen, D. Wu, and A. Li, Appl. Phys. Lett. 105, 052910 (2014).

${ }^{16}$ J. J. Peng, C. Song, B. Cui, F. Li, H. J. Mao, G. Y. Wang, and F. Pan, Appl. Phys. Lett. 107, 182904 (2015).

${ }^{17}$ N. Nakagawa, H. Y. Hwang, and D. A. Muller, Nat. Mater. 5, 204 (2006).

${ }^{18}$ Y. Wang, M. K. Niranjan, K. Janicka, J. P. Velev, M. Ye. Zhuravlev, S. S. Jaswal, and E. Y. Tsymbal, Phys. Rev. B 82, 094114 (2010).
${ }^{19}$ Y.-Z. Wu, H.-S. Lu, T.-Yi. Cai, and S. Ju, AIP Adv. 4, 087109 (2014).

${ }^{20}$ G. Kresse and J. Furthmüller, Phys. Rev. B 54, 11169 (1996).

${ }^{21}$ J. Taylor, H. Guo, and J. Wang, Phys. Rev. B 63, 245407 (2001).

${ }^{22}$ See supplementary material at http://dx.doi.org/10.1063/1.4941805 for the computational details, free-electron tunneling model, ferroelectric displacements, electrostatic potential, and transport results for symmetric FTJs.

${ }^{23}$ As a comparison, we also performed the similar calculations for those symmetric FTJs, as shown in Fig. S1. It can be seen that M-O displacements for the FTJ with $\mathrm{NiO} / \mathrm{BaO}$ interfaces are slightly larger than that of the FTJ with $\mathrm{LaO} / \mathrm{TiO}$ interfaces, which is in line with previous results. ${ }^{19}$

${ }^{24}$ B. Meyer and D. Vanderbilt, Phys. Rev. B 65, 104111 (2002).

${ }^{25}$ M. Gajdoš, K. Hummer, G. Kresse, J. Furthmüller, and F. Bechstedt, Phys. Rev. B 73, 045112 (2006).

${ }^{26} \mathrm{As}$ a comparison, we also calculated the transmission coefficients for those FTJs with symmetric interfaces. Taking the FTJ with $\mathrm{LaO} / \mathrm{TiO}$ at both the left and right interface, for example, the relevant results are shown in Fig. S3. As expected, those symmetric FTJs show the negligible TER effect.

${ }^{27}$ M. Stengel, P. Aguado-Puente, N. A. Spaldin, and J. Junquera, Phys. Rev. B 83, 235112 (2011).

${ }^{28}$ We mention that a sizable TER effect under a finite bias can be achieved even in the FTJ with symmetric electrodes. ${ }^{8}$ This is due to the piezoelectric response of the ferroelectric barrier to an applied bias. In our calculations, the piezoelectric effect of BTO was not taken into account.

${ }^{29}$ K. Momma and F. Izumi, J. Appl. Crystallogr. 44, 1272 (2011). 